
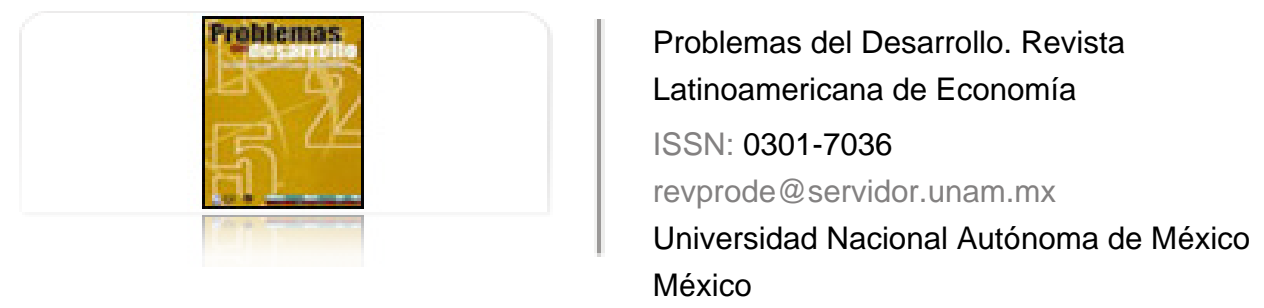

Torres Torres, Felipe; Gasca Zamora, José

El Plan Puebla-Panamá. Una perspectiva del desarrollo regional en el contexto de los procesos de la economía mundial

Problemas del Desarrollo. Revista Latinoamericana de Economía, vol. 35, núm. 138, 2004, pp. 33-56

Universidad Nacional Autónoma de México

Distrito Federal, México

Disponible en: http://www.redalyc.org/articulo.oa?id=11825948003

Cómo citar el artículo

- Número completo

- Más información del artículo

Página de la revista en redalyc.org

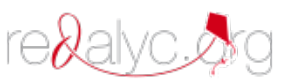

Sistema de Información Científica

Red de Revistas Científicas de América Latina, el Caribe, España y Portugal Proyecto académico sin fines de lucro, desarrollado bajo la iniciativa de acceso abierto 


\section{El Plan Puebla-Panamá. Una Perspectiva del desarrollo REGIONAL EN EL CONTEXTO DE LOS PROCESOS DE LA ECONOMÍA MUNDIAL*}

\section{Felipe Torres Torres ** José Gasca Zamora***}

Fecha de recepción: 2 de abril de 2004. Fecha de aceptación: 25 de agosto de 2004.

\section{Resumen}

Se estudian los esquemas emergentes de politica de desarrollo regional en México. El referente en este caso es el Plan Puebla-Panamá (PPP) que se considera una iniciativa para el desarrollo del Sur-Sureste de México y de articulación con las naciones centroamericanas. En la presente administración se establece que una forma de revertir los desequilibrios es insertando esas regiones rezagadas en los procesos globales mediante la creación de infraestructura, el redireccionamiento de la inversión y el fomento al aprovechamiento de recursos naturales como agua, biodiversidad y energéticos. En esa lógica se orienta la implantación del PPP. El artículo evalúa el impacto y el avance de una iniciativa de esta naturaleza sobre el desarrollo regional y los procesos de integración transterritoriales.

Palabras clave: desarrollo regional, politicas regionales, Plan Puebla-Panamá.

\section{Abstract}

This article looks at the emerging schemes for regional development in Mexico. The reference in this case is the Plan Puebla-Panama (PPP) which is considered as an initiative for the development of Mexico's south-southeast to articulate with the Central American States. In the present Administration it is established that one way of reversing imbalances is by inserting those lagging regions into global processes by creating infrastructure, redirecting investment and promoting the good use of natural resources such as water, biodiversity, and energy. The implementation of the PPP is oriented in line with this. The article assesses the impact and progress of an initiative of this nature on regional development and the processes of crossborder integration.

Key words: regional development, regional policies, Plan Puebla-Panama.

* Este artículo forma parte de los resultados de la investigación: "Territorios en reserva. El Plan Puebla-Panamá y las nuevas estrategias de desar rollo económico regional", que se lleva a cabo con el auspicio de la Dirección General de Asuntos del Personal Académico de la UNAM .

** Investigador del IIEC-UNAM. Correo electrónico: felipet@ servidor.unam.mx

*** Investigador del IIEC-UNAM Correo electrónico: jgasca@servidor.unam.mx 


\section{Résumé}

Les schémas émergeants de politique de développement régional au Mexique sont étudiés. Dans ce cas, on se réfère au Plan Puebla-Panama (PPP), qui est considéré comme une initiative pour le développement du Sud-Sud Est du Mexique et comme une articulation avec les nations centreaméricaines. Sous cette administration, on établit qu'une des façons de retourner les déséquilibres c'est en insérant ces régions retardataires dans les processus globaux au moyen de la création d'une infrastructure, du recyclage de l'investissement et d'encourager l'utilisation des ressources naturelles telles que l'eau, la biodiversité et les énergétiques. C'est dans cette logique que le PPP est orienté. L'article évalue l'impact et le progrès d'une initiative de cette nature sur le développement régional et sur les processus d'intégration transterritoriaux.

Mots clés: développement régional, politiques régionales, Plan Puebla-Panama.

\section{Resumo}

Um estudo dos modelos emergentes da politica do desenvolvimento regional no México, a partir de uma análise do Plano Puebla-Panamá (PPP), que é considerado uma iniciativa de desenvolvimento do Sul-Sudeste do México e de articulação com as nações centro-americanas. Para a atual administração, a melhor forma de reverter os desequilibrios é inserir regiões pouco desenvolvidas nos processos globais, mediante a criação de infra-estrutura, redirecionamento dos investimentos e estímulo ao aproveitamento dos recursos naturais - como a água, a biodiversidade e os energéticos. Esse pensamento sugere a implantação do PPP. O artigo avalia o impacto e as vantagens de uma iniciativa desta natureza sobre o desenvolvimento regional e sobre os processos de integração transterritoriais.

Palavras-chaves: desenvolvimento regional, políticas regionais, Plano Puebla-Panamá. 


\section{Introducción}

$\mathrm{D}$ esde mediados del siglo xx se ha insistido, dentro de las políticas públicas en diciones que tiendan al equilibrio y a la integración territorial del país. Con el tiempo, esta consideración ha sido importante en la medida que en otros países se ha probado que es un medio que contribuye a mejorar la integración del aparato productivo, detona las capacidades productivas de las regiones y genera empleo y arraigo poblacional.

Sin embargo, la mayoría de las políticas de corte regional implantadas en México en décadas pasadas tuvieron impactos aislados y coyunturales que las alejaron de sus propósitos originales. Las inercias negativas que propició el modelo interno de economía protegida se reflejaron en estrategias que sobreexplotaban los recursos de las regiones, no diversificaban ni otorgaban continuidad a las inversiones y, finalmente, generaron empobrecimiento gradual y una creciente marginación de algunas regiones del país.

Hoy se reconoce que en el contexto de apertura económica no basta con instrumentar políticas macroeconómicas para generar desarrollo, sino que se necesitan estrategias regionales que favorezcan los niveles de integración del mercado nacional y la creación de condiciones de competitividad para participar en los circuitos de comercio e inversión a escala mundial.

La propuesta de desarrollo regional del presente sexenio busca establecer estrategias adaptadas al entorno mundial mediante la incorporación de regiones que han tenido un papel marginal en los procesos de desarrollo que ha experimentado el país. Estas regiones han sido revaloradas debido a la presencia de recursos de un alto potencial y a las ventajas de localización para inversiones productivas internacionales.

Un tipo de política regional de esta naturaleza es el denominado Plan Puebla-Panamá (PPP), que se centra en los estados menos desarrollados del país, ubicados en el Sur y Sureste de México. Este plan incorpora, al mismo tiempo, una estrategia de carácter transfronterizo, pues engloba los siete países que conforman el área centroamericana.

La situación de atraso y la base económica limitada en las diversas áreas que integran el conjunto de la región del pPP plantean el siguiente dilema: recibir una inyección temporal de apoyos financieros dirigidos hacia la explotación de recursos valiosos o quedar nuevamente marginados de los procesos de inversión y acumulación de capital.

En este contexto, los gobiernos de los distintos países involucrados también enfrentan el dilema de cómo contener los flujos migratorios — resultado de la pobreza- hacia el Norte, junto con la crisis o el deterioro de sus regiones menos desarrolladas. Lograr el equilibrio

\section{Déparrersollo}


entre crecimiento económico, expansión del capital y desarrollo regional se convierte así en uno de los problemas por resolver en países como México y las naciones centroamericanas.

A la luz de esta situación, en este trabajo nos proponemos discernir si un esquema de desarrollo, como el propuesto por el PPP — con alta complejidad para inducir las regiones marginadas a los mercados mundiales - se puede traducir en procesos que irradien beneficios en las regiones. Más aún, si el Estado, mediante políticas públicas como ésta, se encuentra en condiciones de disminuir las desigualdades y generar procesos amplios y diversificados que puedan ser detonantes de desarrollo regional.

El documento se compone de cinco apartados. En la primera parte se hace una revisión de las modalidades teóricas que han asumido las políticas de desarrollo regional. En el segundo apartado se realiza una evaluación de dichas políticas implantadas en nuestro país, su alcance e incidencia en los procesos de estructuración territorial. En tercer lugar, se trata de plantear el PPP como ejemplo de los procesos de inversión-comercio a escala mundial. En la cuarta sección, y a tres años de su inicio, se procede a identificar el avance del Plan Puebla-Panamá, en términos de las áreas hacia las cuales se ha orientado el financiamiento y los proyectos instrumentados. Finalmente, se lleva a cabo un análisis del componente transnacional del PPP, a partir de los mecanismos de integración y proyectos compartidos entre México y las naciones centroamericanas adscritas al Plan.

\section{Las politicas contemporáneas de desarrollo regional: una revisión de sus propuestas teóricas}

A partir del problema de las desigualdades regionales y la polarización territorial en el marco del desarrollo capitalista, se reconoció la necesidad de instrumentar acciones y medidas compensatorias para corregir dicha problemática. Ello tenía como finalidad garantizar un crecimiento cohesionado de la economía nacional, poniendo énfasis en el apoyo a los territorios más atrasados. Éste fue el principio que condujo al surgimiento de políticas regionales, es decir, el conjunto de medidas destinadas a reducir los desequilibrios interregionales. El concepto también ha sido entendido en el sentido de promoción del desarrollo regional, esto es, esfuerzos al interior de las regiones para superar los problemas de atraso, declive económico, crisis o desempeño, lo que supone que el mencionado desarrollo no solamente está relacionado con regiones atrasadas (Pujadas y Font, 1998:53).

Las políticas regionales ocuparon un lugar en las agendas de desarrollo de diversos estados nacionales desde la década de los treinta del siglo pasado y a partir de la segunda posguerra. El Estado se erigió como la instancia capaz de instrumentar procesos compensatorios, ordenadores o conductores de las regiones y sus formas de integración a los proyectos nacionales, legitimando con ello un orden social territorial. Desde esta perspectiva, se convierte en el referente principal de los procesos de desarrollo y de organización territo- 
rial, y el punto de partida para comprender los estilos en la forma de concebir y hacer política regional.

Las formas de intervención estatal en su vertiente regional y territorial no se limitaron a políticas regionales, sino a un paquete de acciones e instrumentos que les dieran sentido. La planeación y la gestión territorial y regional legitimaron formas concretas de intervención y regulación estatales, expresadas en la definición de regionalizaciones, marcos legales, instituciones, programas y proyectos. En conjunto, estos instrumentos tenían el propósito de incidir en procesos deseables de organización territorial y desarrollo regional.

Aunque las políticas regionales y sus instrumentos han variado de acuerdo con cada país, podemos identificar modelos que muestran rasgos comunes a lo largo del tiempo, en términos de los esquemas de intervención estatal, de lo cual se desprenden formas específicas en la manera de definir e institucionalizar estilos de desarrollo y políticas públicas en este campo.

De acuerdo con Bert Helmsing (1999), a partir de la segunda mitad del siglo xx se pueden identificar tres generaciones en la forma de concebir y abordar el problema del desarrollo regional mediante políticas y estrategias. La primera generación surge entre las décadas de los cuarenta y cincuenta. El tema central era el crecimiento económico, pues al ser desigual y selectivo en diversas partes del territorio, se creaban desigualdades regionales. En términos de interpretación, surgieron distintas apreciaciones entre quienes señalaban que las desigualdades se incrementarían con el paso del tiempo y, por el contrario, quienes postulaban que el desarrollo del mercado tendía a generar equilibrios y procesos de convergencia regionales.

Una forma de resolver este problema consideró el enfoque keynesiano como un referente para establecer mediaciones desde el Estado en los procesos de desarrollo. A partir de ello se definió un estilo basado en políticas públicas orientadas a reducir los impedimentos de la movilidad de los recursos y a transferir elementos monopólicos que mantuvieran los precios fuera de sus niveles competitivos. El Estado fue, entonces, el actor central de políticas subsidiarias y compensatorias a escala regional y generó, al mismo tiempo, las condiciones necesarias para el proceso de acumulación del capital.

Las modalidades que asumió la política regional de corte keynesiano en diversos países se orientaron en varios sentidos. Se privilegió la concentración de recursos en grandes proyectos seleccionados y se impulsó la creación de polos de desarrollo o crecimiento. Para estos casos, se otorgó prioridad al sector industrial mediante parques, ciudades y corredores industriales, y se establecieron mecanismos de compensación hacia regiones atrasadas y en los que se promovió la atracción de inversiones foráneas, entre otros.

Los instrumentos para facilitar estos procesos fueron variados: incentivos fiscales y financieros, precios preferenciales, energéticos baratos, expropiación y acceso a terrenos y dotación de infraestructura, y otros. Estos factores, justamente buscaban incidir en deter- 
minada localización de empresas y estimular la demanda local para reducir las desventajas regionales.

Durante la década de los setenta y ochenta tuvieron lugar distintos procesos que obstaculizaron el desempeño de las políticas de Estado orientadas a la regulación estatal y la corrección de las desigualdades regionales, tal como venían desempeñándose. Las crisis fiscales - recurrentes en diversos países - y el nuevo entorno mundial promovían la apertura de las economías nacionales y procesos de desregulación estatal y reestructuración productiva y territorial.

Fueron inicialmente algunos países industrializados quienes descubrieron y formularon estrategias de desarrollo regional no ortodoxas, a partir de los procesos de reestructuración territorial que venían ocurriendo en esas mismas naciones. El fenómeno observado fue un declive para sus regiones tradicionales frente a otras que tenían capacidad para competir en el mercado internacional. Estas regiones emergentes, ubicadas principalmente en Europa y Estados Unidos, denominadas nuevos distritos industriales, se consideran ejemplos paradigmáticos en un nuevo esquema de desarrollo regional industrial. Dicho esquema está basado en capacidades locales de innovación que permiten el desarrollo de mercados endógenos y el mejoramiento de las condiciones para competir en mercados internacionales.

Partiendo de este hecho, Krugman considera que el comercio, la especialización, las economías de escala y la competencia imperfecta resultan más importantes que los rendimientos decrecientes, la competencia perfecta y la ventaja comparativa, ya que las economías externas basadas en tamaño de mercado e innovación tecnológica que apuntalan a ésta, no son de alcance nacional o internacional, sino que surgen de un proceso de aglomeración regional o local (Moncayo, 2002).

Las posibilidades que brindan los procesos de flexibilización a escala territorial y su expresión en forma de distritos industriales son consideradas un elemento cualitativo de la segunda generación de políticas de desarrollo regional. A diferencia de la primera fase, el Estado ya no representa el centro de conducción política y económica. El desarrollo se consigue fomentando la cooperación entre diversos actores, tales como empresas, gremios industriales y sindicatos, además de acciones del Estado, reducidas a mecanismos de gestión y fomento de las instituciones gubernamentales. Con ello, se espera desarrollar un conjunto de habilidades, recursos y conocimiento de la región y la comunidad regional (Helmsing, op. cit).

A partir de lo anterior, en el inicio de la década de los noventa, el conocimiento de los factores de promoción del desarrollo regional se sistematizó y con base en las experiencias que mostraban varios países como Italia, Alemania, Japón, Inglaterra, Francia y Estados Unidos. En este sentido, se propuso una nueva vertiente de estrategias de desarrollo regional que considera la importancia de sinergias, capacidades y conocimiento como detonadores de procesos de desarrollo regional endógeno. Desde este enfoque se valora el ámbito mundial como escenario de competencia y, por ello, las políticas no sólo se restringen a 
ámbitos locales, sino que consideran la ubicación y el posicionamiento de sistemas de producción regionales en el contexto global.

Las políticas regionales más recientes, enmarcadas en una tercera generación, consideran que no se requiere invertir más recursos desde el Estado, sino aumentar la racionalidad sistémica en el uso de recursos y programas existentes. Este enfoque supera la oposición entre políticas de desarrollo endógeno y exógeno, porque presupone mecanismos de complementariedad y articulación entre los actores (Estado-sector privado-sociedad) y las escalas de acción: local, regional, nacional e internacional (Helmsing, op. cit.).

Estas políticas de desarrollo regional se han diseñado con un enfoque neoclásico del crecimiento económico, y parten del supuesto de que el libre juego de las fuerzas del mercado conduce a los países y sus regiones a igualar sus niveles de desarrollo, convirtiendo en innecesarias las políticas intervensionistas al estilo del enfoque keynesiano que modeló la primera generación de políticas regionales (Moncayo, op. cit.).

En este escenario, según Porter, el Estado debe proporcionar el marco para un entorno favorable, es decir, actuar como agente promotor, responsable de dotar al país de infraestructura básica, desarrollo tecnológico y servicios de educación y salud, para permitir a las empresas competir con ventaja en el mercado internacional. El análisis de nuevas situaciones, la gestión de visiones compartidas y el esfuerzo conjunto de los actores del desarrollo regional, aunado al papel facilitador y promotor del desarrollo, constituyen el verdadero reto de la competitividad regional, en cuanto a la forma de insertarse en la economía mundial (Indacochea, 2001).

A diferencia del pasado, la competitividad ya no depende sólo de los recursos naturales o de la mano de obra barata, sino del capital humano que induce innovaciones para diseñar, producir o comercializar bienes y servicios de mayor calidad o a menor precio que la competencia internacional. A pesar de que ésta se ha mundializado, las economías locales son cada vez más determinantes para la competitividad. Las regiones que ganan se caracterizan por contar con una estrategia de inserción en la economía global, un tamaño adecuado para la innovación, infraestructura de comunicaciones y red de servicios que permita considerarlos como una red de negocios en la economía mundial.

La necesidad de un nuevo tipo de acción estatal corresponde a las presiones de revaloración del territorio, direccionadas por la reestructuración económica mundial que, a partir de nuevas formas de acumulación, busca mayor flexibilidad, tanto en los procesos de producción y gestión empresarial, como en los mercados de trabajo, a fin de facilitar la calificación de los recursos humanos. En la medida en que el nuevo modelo de acumulación se basa en el acceso al conocimiento y en la información estratégica, obliga a definir prioridades en la calidad de dichos recursos humanos para que sostengan los procesos productivos (Alburquerque, 2001).

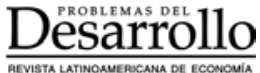


Para situarse en perspectiva ganadora o alcanzar los nuevos niveles de valorización, el territorio debe ofrecer ventajas que lleven a optimizar la interconexión de los capitales mundiales, es decir, garantizar infraestructura o perspectiva de uso de los recursos para que éstos se conviertan en factor de promoción y atracción de capital externo. La visión exógena por la vía de la inversión, aún promoviendo el desarrollo endógeno, es la fuente del desarrollo regional actual.

\section{Las politicas de desarrollo regional de México y su incidencia en los procesos de estructuración territorial}

El desarrollo económico integral de la nación constituye un supuesto básico de las políticas de gobierno que se han emprendido en México. Su aplicación se ha circunscrito a programas predominantemente macroeconómicos, apoyados en la promoción de estrategias sectoriales claramente identificadas en los planes de gobierno prevalecientes. Si bien esta forma de considerar el desarrollo económico ha servido para el impulso de determinados sectores, en la dinámica del desarrollo regional y el beneficio social del conjunto de la población ha generado efectos opuestos, pues las ventajas quedan contrarrestadas por los impactos derivados de las asimetrías regionales. Ante tal evidencia, las políticas regionales en México han sido medidas que amortiguan, en parte, la ausencia de una política integral de desarrollo que tome en cuenta la condiciones, capacidades y necesidades de los territorios.

A pesar de dicho sesgo, a lo largo del siglo pasado, en nuestro país hubo esfuerzos importantes que mostraron un contenido implícito o explícito en cuanto a su orientación regional. Desde la década de los cuarenta hasta mediados de los años ochenta, las políticas regionales se diseñaron con el esquema de economía cerrada y en la lógica de que los procesos de acumulación y reproducción del capital estaban comandados por el Estado.

Desde un marco de centralismo y presidencialismo exacerbado se promovieron la industrialización por sustitución de importaciones y los procesos de inversión y promoción estatales que incluían la creación de infraestructura económica, exención de impuestos y abastecimiento de energéticos baratos, entre otros. Ello significaba esquemas privilegiados o compensatorios hacia distintas regiones y ciudades.

En esta etapa se crearon leyes y programas de impulso al desarrollo industrial (Leyes de Exención Fiscal, 1940; Leyes de Industrias Nuevas y Necesarias, 1941-1955; Programas de Parques y Ciudades Industriales, 1960-1970). La construcción de infraestructura física, principalmente redes de transporte y equipamiento urbano, permitió la interconexión del incipiente sistema de ciudades y la consolidación de zonas urbanas.

En este sentido, la capital del país y las entidades del Centro se fortalecieron como el núcleo demográfico de la nación y el eje de desarrollo industrial. Es justamente en la llamada corona megapolitana donde se ha fortalecido un sistema urbano altamente integrado a la ciudad de México mediante las redes de ciudades medias que la circundan:

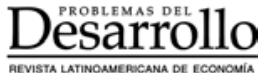


Pachuca, Toluca, Cuernavaca, Tlaxcala y Puebla, las cuales desempeñan funciones de pivote para el desarrollo manufacturero y para la circulación de mercancías entre el Centro y el resto del territorio nacional.

Durante la década de los setenta y principios de los ochenta, se institucionalizaron esquemas que permitieron otorgarle un cierto orden y sentido al desarrollo regional y territorial de nuestro país. Además, se llevaron a cabo diversas acciones aisladas o coyunturales para promover el desarrollo de regiones, mediante planes y programas como por ejemplo, para las zonas áridas (1970), el Istmo de Tehuantepec (1972), las zonas marginadas (1977), La Chontalpa y la frontera norte, entre otros. En esta fase se logró integrar la primera Ley de Asentamientos Humanos (1977), la cual le otorgó una nueva dimensión a los procesos de ocupación y regulación del territorio, y generó el primer Sistema Nacional de Planeación, a partir del cual se incorporaron las entidades federativas y los municipios a los procesos de gestión de desarrollo regional en sus respectivos ámbitos políticoadministrativos.

Aunque hubo avances importantes en la creación de los sistemas de planeación territorial y regional, hasta el momento los esfuerzos por crear un soporte institucional sólido y articulado han tenido limitaciones. Por ello, es necesario identificar los diferentes niveles y el significado de las políticas con un sentido regional implícito o explícito.

De acuerdo con Ferreira (2003), existen cuatro modalidades en las cuales se expresan las políticas regionales y cada una responde a objetivos específicos. Una política de desarrollo, esto es, el marco de referencia global respecto del desarrollo nacional, susceptible de guiar los patrones de desarrollo territorial y los programas regionales. Una política regional nacional orientada a articular estrategias de desarrollo e integración de grandes territorios (en el caso de nuestro país esto se reduce a un programa de ordenamiento territorial y urbano). Políticas regionales que promuevan proyectos interestatales y esquemas de articulación territorial. Planes y programas de carácter estatal y municipal que busquen orientar los modelos de desarrollo y coordinar los procesos de inversión hacia estas unidades político-administrativas.

Desde mediados de la década de los ochenta, nuestro país transita hacia un modelo de apertura. En este esquema se focaliza el interés hacia el comercio exterior, el desarrollo de maquiladoras y ciertas ramas exportadoras. En este periodo comienza una nueva generación de políticas públicas que dan lugar a diversos procesos de desregulación de nuestra economía, y se fomenta una mayor apertura a las inversiones extranjeras directas, consideradas como una de las principales fuentes para la reactivación de ciertos sectores y regiones.

Desde este esquema de desarrollo exógeno, el Estado deja de ser el principal vertebrador de los procesos territoriales y de desarrollo regional. En esta fase, las expresiones territoriales del desarrollo se orientan hacia la consolidación de nuevos procesos urbano-industriales, la generación de infraestructura productiva y la consolidación de plataformas exportadoras

\section{Déparrersollo}


en ciudades medias ubicadas en las regiones del Centro-Occidente, el Norte del país y la frontera con Estados Unidos de América. Como resultado de esto, se configura un modelo territorial más descentralizado (a diferencia del anterior) y emerge un nuevo espacio de desarrollo hacia las entidades del Centro-Occidente y Norte del país. La vitalidad en estas regiones ha estado marcada por el emplazamiento de sectores manufactureros dinámicos, vinculados a inversiones transnacionales y del mercado externo. Éste sería el caso de ciudades como Guadalajara, Querétaro, Aguascalientes y San Luis Potosí.

En términos de políticas de carácter regional, en las últimas dos décadas han predominado tres modalidades. Una de ellas se podría llamar enfoque regional asistencialista, representado por programas de gasto social dirigidos hacia grupos identificados en pobreza extrema o con alto grado de marginación. Éste es el caso de los programas regionales de solidaridad implantados durante la administración de Salinas y los programas de microrregiones de las administraciones de Zedillo y Fox. Otra orientación corresponde a los programas dirigidos a consolidar o mejorar el posicionamiento de centros urbanos mediante equipamiento e infraestructura física. Los ejemplos para este caso fueron los diversos programas de desarrollo urbano, como el de 100 ciudades medias, que tuvieron vigencia durante el sexenio de Salinas, y los programas de Desarrollo Urbano y Ordenamiento Territorial con los últimos dos mandatarios.

El tercer enfoque corresponde a los programas y proyectos para crear y ampliar infraestructura productiva en grandes espacios regionales, por medio de redes de transporte y comunicaciones: carreteras, puertos, aeropuertos, hidroeléctricas y redes de fibra óptica, principalmente. En programas como la Escalera Náutica del Mar de Cortés, el Programa de Desarrollo Regional de la Frontera Norte, el Proyecto de Gran Visión para el Centro del País y el Plan Puebla-Panamá, instrumentados en la presente administración, se considera la importancia que tiene la generación de infraestructura productiva como factor de competitividad, pues incrementa la conectividad de los mercados y reduce los costes de movilidad de las mercancías. También puede contribuir a atraer inversiones foráneas, detonar actividades económicas y posibilitar el aprovechamiento de recursos endógenos.

La justificación para la promoción de nuevas estrategias de desarrollo regional, como las plasmadas en el Plan Puebla-Panamá, parten del supuesto de que el modelo de economía cerrada - como el que prevaleció en México durante el periodo de sustitución de importaciones - causó desequilibrios regionales a partir de la década de los setenta del siglo pasado, ya que privilegió el enfoque sectorial y no el territorial, distorsionó el efecto de los subsidios gubernamentales para el desarrollo al encauzarlo a programas sociales y provocó, de todas formas, un incremento de la pobreza. En contraposición, se afirma que estrategias acordes con el modelo de economía abierta pueden compensar tales desequilibrios.

A partir de la administración del presidente Fox, se establece que una forma de revertir esos efectos es insertando las regiones rezagadas de nuestro país en los procesos globales

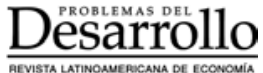


mediante la creación de infraestructura; el redireccionamiento de la inversión para el aprovechamiento de recursos naturales en espacios con agua, biodiversidad y energéticos; su posición geográfica estratégica en los esquemas de circulación y distribución de mercancías; la promoción de mayores flujos de inversión productiva para incrementar el ingreso; el mejoramiento de la calidad de vida; el fomento al arraigo regional y la contención de los flujos migratorios hacia el norte. En esa lógica se orienta la implantación del Plan Puebla-Panamá, el cual recupera la política regional como una modalidad del desarrollo económico interno, tomando en consideración la dinámica que imponen los procesos globales de la economía.

\section{El Plan Puebla-Panamá: un caso de incorporación de los territorios en las estrategias globales de inversión y comercio}

El Plan Puebla-Panamá ha revitalizado expectativas de políticas de carácter regional que además rompen con las formas ortodoxas de la planeación regional en México y generan muchas interrogantes, inquietudes teóricas y diversas posiciones sobre sus posibles impactos en el desarrollo económico y social de las regiones.

El tipo de desarrollo regional que se busca actualmente para México se ubica en el marco de una sucesión de eventos que no pueden considerarse recientes, menos como expresión de una nueva reconfiguración territorial interna. Responde también a las lógicas que persigue el capital productivo y financiero (mediante empresas y consorcios financieros, a escala mundial) por incorporar espacios que habían permanecido como reservas territoriales en determinados países y que se convierten, por su localización estratégica y la disponibilidad de recursos, en sitios atractivos para la inversión, el tráfico mundial de mercancías y su incorporación a los mercados globales en ventajas estratégicas para la competencia por el control de los mercados.

La concepción del PPP como proyecto de desarrollo regional en el contexto de la globalización ocurre en el marco de contradicciones regionales alimentadas por rezagos y conflictos que se canalizan hacia la necesaria defensa de los recursos naturales y de las identidades culturales, particularmente entre las comunidades indígenas con fuerte presencia local. Los antecedentes para su incorporación a un nuevo tipo de desarrollo regional global mediante el PPP se remontan al diagnóstico derivado de una visión de corte neoclásico del uso del espacio en condiciones de atraso, del cual se considera que se puede alcanzar la homogeneidad en los procesos económicos del territorio. El documento denominado El Sur También Existe, reconocido como el principal antecedente del PPP, plantea que las políticas instrumentadas por el Gobierno Federal han desalentado el desarrollo económico de Chiapas y de la región sureste. Se considera factible complementar las acciones de política social mediante una conexión de los factores que han reprimido el aprovechamiento de las ventajas comparativas naturales del sureste. Al parecer, las políti-

\section{Déparrersollo}


cas públicas han afectado la distribución espacial de la producción y el patrón de ventajas comparativas entre las distintas zonas del país, según indicadores de la política del gasto público en infraestructura de transporte, en política de precios y tarifas del sector público, así como en el régimen de comercio exterior (Dávila, Kessel y Levy, 2002).

En dicho trabajo también se establece que la política de desarrollo regional no debe plantearse un crecimiento igual en todas las zonas del país, sino mejorar sus posibilidades de desarrollo. La apertura comercial implica un cambio en la distribución geográfica de la producción, lo cual requiere un proceso de adaptación de tasas de crecimiento distintas para las diversas regiones. La nueva estrategia, en el marco del TLCAN, la apertura comercial y la globalización necesitan un incremento en las inversiones públicas para infraestructura de transporte y desarrollo de hidroeléctricas, junto con cambios institucionales regulatorios, sobre todo en política de precios, tarifas y subsidios.

El diagnóstico gubernamental que justifica al PPP, además, reconoce que, en general, las iniciativas regionales son desarticuladas y discontinuas, y tienen resultados disímbolos y poco eficaces frente a las fuerzas económicas, territoriales y sociales a las cuales trata de orientar. En ese contexto, por su inaccesibilidad a los centros dinámicos del país y debido a la carencia de conexiones directas de cierta envergadura con los mercados internacionales, la región Sur-Sureste ha vivido procesos económicos de cierta autonomía, pero de escala reducida. Las excepciones fueron el turismo y la actividad petrolera, más en un esquema de enclave que como motores de desarrollo regional integrado (la migración ha sido un mecanismo para sortear la falta de oportunidades en estos espacios).

Uno de los supuestos teóricos del pPP es que todas las economías se benefician de una mayor integración de los mercados internacionales y así, las de escaso capital y costos más bajos, tienen más posibilidades de beneficiarse con una mayor integración, que aquellas otras con abundancia de capital y costos más elevados.

El objetivo de impulsar un tipo inédito de desarrollo regional transfronterizo no responde necesariamente sólo a la gestión de los gobiernos de México ni de los países que conforman la región centroamericana; tampoco es competencia de un solo gobierno local, sino que obedece a un cúmulo de intereses externos históricos que requieren ahora del posicionamiento en esos territorios para relanzarlos hacia distintas vertientes del desarrollo y lograr, tanto la refuncionalización, como la competitividad de los capitales locales e internacionales.

La región Sur-Sureste, según el diagnóstico oficial, no es un espacio homogéneamente pobre o marginado. Comprende polos o zonas de dinamismo económico que no han influido en el desarrollo de la región. La estrategia territorial del ppp identifica grandes espacios subregionales, relativamente poco articulados entre sí con un alto potencial entre los actores correspondientes: Istmo de Tehuantepec, arco del Golfo de México, el Mundo Maya (península de Yucatán, Chiapas y países centroamericanos fronterizos) y el Sur. Un 
objetivo tiene que ver con la conectividad (telecomunicaciones y carreteras), y el segundo consiste en el fortalecimiento de las relaciones entre las subregiones.

Se parte de que la región no tiene un referente de articulación ni un modelo bien definido de estructuración y funcionamiento urbanos. Tampoco cuenta con un espacio de intercambio tan cercano e intenso, como sería el caso de la región Norte del país con respecto de Estados Unidos de América. Se concluye, entonces, que la región del pPP se caracteriza por un patrón de desarrollo urbano básicamente costero, complementado por centros administrativos estatales (Oaxaca, Chilpancingo, Villahermosa y Tuxtla Gutiérrez), polos de explotación de hidrocarburos y gas (Coatzacoalcos, Ciudad del Carmen, Cactus) y ciudades dinamizadas por el turismo (Cancún, Acapulco, Huatulco).

Un rasgo dominante en la instrumentación de políticas públicas de desarrollo, como las que derivan del PPP, es el espacio de mediación que desempeñan los estados nacionales porque asigna papeles diferenciados a los agentes involucrados en escalas menores. En el plano nacional, el Estado asume la posición de promotor y facilitador para generar ambientes de negocios favorables que permitan aterrizar las estrategias internacionales de inversión-comercio. Para ello, fija los parámetros del desarrollo y promueve la creación de agencias y alianzas intergubernamentales e interregionales para el desarrollo. En la escala regional subnacional, los gobiernos estatales hacen lo propio, asumiendo también funciones activas de marketing y paradiplomacia regional para ganar las mayores inversiones hacia sus espacios; pero además, se convierten en socios instrumentadores de la estrategia definida en la agenda general, ya que es justamente en la escala regional y local donde la aplicación de proyectos asume su verdadero significado.

\section{El Plan Puebla-Panamá, el camino recorrido en tres años}

La iniciativa del PpP fue publicada oficialmente en México por el Gobierno Federal el 12 de marzo de 2001. En tanto iniciativa transfronteriza, fue consensada tres meses después entre los gobiernos de México y los de los países centroamericanos como parte del Mecanismo de Tuxtla durante la reunión de presidentes en junio de 2001.

En la estructura de organización y funcionamiento del PPP existen actores claves para la orientación y desarrollo de la iniciativa. En primer lugar, México, por medio del Gobierno Federal, se mantiene como una instancia mediadora y desempeña un liderazgo importante en la agenda principal del Plan. En su vertiente centroamericana, un segundo actor es el llamado Grupo Técnico Interinstitucional (GTI), conformado por representantes del BID, el BCIE, la CEPAL. Dicha instancia se ha encargado de definir la conectividad de los proyectos del PPP a partir de junio de 2001.

El diagnóstico inicial del PPP, elaborado por el Gobierno Federal, se realizó mediante un modelo de planeación estratégica que permite ubicar potencialidades y riesgos a partir de matrices llamadas FODA que identifican: fortalezas, oportunidades, debilidades y ame-

\section{DeSRarrollo}


nazas. Las ventajas comparativas detectadas por FODA se pueden resumir en tres aspectos: potencial productivo, basado en recursos naturales y energéticos (biodiversidad, agua, petróleo y electricidad); mercados de trabajo de baja remuneración, y ventajas de localización en los procesos hemisféricos y mundiales de circulación de mercancías.

El programa propuesto por el PPP da la idea de que "el desarrollo es primordialmente de carácter humano" (Presidencia de la República, 2001a), por lo que propone acciones de lucha contra la pobreza, promoción de inversiones productivas y creación de infraestructura y sustentabilidad ambiental. El Documento Base del Capítulo México (Presidencia de la República, 2002) define como objetivo principal: mejorar la calidad de vida de los habitantes del Sur-Sureste. A la vez, considera ocho objetivos orientados a lo siguiente: desarrollo social y humano, participación de la sociedad civil en el desarrollo, cambio estructural en la dinámica económica de la región, aprovechamiento de vocaciones y ventajas comparativas, manejo sustentable de recursos naturales y el ambiente, concertación de planes y estrategias conjuntas entre el Sur-Sureste y los países de Centroamérica, modernización y fortalecimiento de la capacidad de las instituciones de este espacio.

La región, identificada como el Sur-Sureste por el Plan Nacional de Desarrollo, considera nueve entidades: Puebla, Guerrero, Oaxaca, Veracruz, Tabasco, Chiapas, Campeche, Yucatán y Quintana Roo. El territorio comprendido por dichas entidades alberga a $28.2 \%$ de la población nacional y a 72\% de la población indígena, pero sólo genera $17.5 \%$ del PIB nacional. En promedio, estas entidades tienen un ingreso per capita $50 \%$ menor al de los estados del Norte del país.

Los indicadores de pobreza, tales como mortalidad infantil, analfabetismo y población en localidades aisladas, con alta y muy alta marginación, muestran un fuerte contraste con respecto de los valores registrados en las entidades más desarrolladas. Estas diferencias reflejan el fuerte rezago del desarrollo socioeconómico del s-SE en relación con las regiones del Centro y Norte (Presidencia de la República, 2001). Como casos extremos, Oaxaca y Chiapas tienen más de 50\% de su población en localidades clasificadas de alta o muy alta marginación; Guerrero, 45\%; Puebla y Veracruz, más de 30\%; Yucatán y Campeche, entre 20 y 30\%; mientras que Quintana Roo y Tabasco, entre 10 y $20 \%$ (Rudiño, 2001). Los indicadores económicos y sociales promedio de esta región son más parecidos a los de algunas naciones centroamericanas que a las entidades del Centro o Norte de nuestro país.

Dentro de los objetivos del PPP, y en la lógica de las ventajas comparativas consideradas por las matrices de FODA para la región S-SE, se plantea un conjunto de estrategias integradas a una cartera de proyectos en diferentes renglones. Sin embargo, en la escala de prioridades no fue el desarrollo social y humano lo que se privilegió, tal como lo plantea el primer objetivo del PPP, sino los proyectos de infraestructura de transporte, hidroagrícola y de energía, pues se infiere que éstas son las áreas más importantes para atraer o detonar inversiones en distintos lugares de la región.

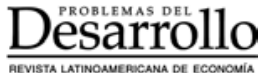


La definición de los renglones estratégicos del plan también presenta como propósito negociar recursos federales, a fin de integrar un programa de inversión. En este sentido, quedó establecida la partida PPP, incluida por primera vez en el Programa de Egresos de la Federación de 2002. Para esta partida se asignaron 7457.1 millones de pesos, de los cuales casi $84 \%$ se orientaron a proyectos de la Secretaría de Comunicaciones y Transportes, mientras que los renglones de proyectos productivos, desarrollo social y salud apenas tuvieron recursos equivalentes a menos de $5 \%$ del total. Entre estos últimos se consideraron 50 millones de pesos para estudios y proyectos integrales y algunos programas sociales propuestos por los estados, como el de Medicina Tradicional en Puebla (25.4 millones de pesos) y Albergues Escolares Indígenas en Guerrero (56 millones de pesos).

Cuadro 1

Presupuesto de Egresos de la Federación Recursos destinados al PPP en 2002

\begin{tabular}{lcc}
\hline \multicolumn{1}{c}{ Dependencia } & Millones de pesos & Porcentaje \\
\hline Presidencia de la República & 13.2 & 0.17 \\
Comunicaciones y Transportes & 6251.8 & 83.8 \\
Economía & 107.1 & 1.45 \\
Medio Ambiente y Recursos Naturales & 325.1 & 4.36 \\
Desarrollo Social & 177.2 & 2.37 \\
Turismo & 507.7 & 6.81 \\
Salud & 25.0 & 0.33 \\
Proyectos integrales del Plan & 50.0 & 0.67 \\
& & \\
Total 1/ & 7457.1 & 100.00 \\
\hline
\end{tabular}

1/ Incluye gasto directo, ayudas, subsidios, transferencias, recursos propios de las entidades de control presupuestario indirecto de sct y Turismo.

Fuente: Presidencia de la República. Programa de Inversiones 2002. Plan Puebla-Panamá.

En el desglose presupuestal, la creación, reactivación o modernización de nueva infraestructura para el transporte (carreteras, puertos y aeropuertos) conforman la columna vertebral. Esto se explica por el papel fundamental que se le otorga a la creación de infraestructura física en la refuncionalización económica de los territorios considerados y el desarrollo de nuevas capacidades, orientadas a los circuitos de inversión. La apuesta es que las nuevas empresas se sientan atraídas por la infraestructura y equipamiento creado para conectarse y acceder competitivamente al mercado nacional e internacional.

Entre los proyectos de infraestructura de transporte considerados por el PPP destacan los corredores del Golfo, Pacífico y Transísmico, mismos que podrían reactivarse con la renovación, aumento o construcción de 16 ejes carreteros. El proyecto incluye la modernización del ferrocarril del Sureste, el Chiapas Mayab y del Istmo de Tehuantepec y la restauración o ampliación de las terminales de Terán y Tuxtla Gutiérrez, al igual que los 
Puertos de Coatzacoalcos, Salina Cruz, Dos Bocas y Puerto Madero (Secretaría de Comunicaciones y Transportes, 2001).

En la redefinición y vinculación con el conjunto de proyectos y corredores nacionales, el s-SE propone articulaciones con tres grandes corredores interregionales que, a su vez, estarían orientados al impulso de sectores específicos de la economía:

1. Corredor Veracruz-Monterrey. Corredor Multimodal, logístico y energético del Golfo de México que articula Tabasco y Chiapas hacia la frontera México-Estados Unidos.

2. Circuito peninsular de Yucatán. Corredor turístico que inicia en Cancún-Riviera Maya y de aprovechamiento de la biodiversidad de la península de Yucatán y el Caribe.

3. Corredores transversales. Corredores logísticos manufactureros, maquiladores, turísticos y de aprovechamiento de la biodiversidad, integrados a su vez por el llamado corredor Milenio (Veracruz-Acapulco), el Acapulco-Tuxpan, el del Altiplano Central y Circuito Transísmico de Tehuantepec.

En 2002 se reformuló la concepción original del PPP, principalmente en lo que respecta a las formas de articulación, participación y definición de atribuciones entre el Gobierno Federal de México con los países centroamericanos y las entidades del Sur-Sureste de México. A partir de un nuevo documento, se incorporan mecanismos operativos para los procesos de gestión y planeación en diferentes escalas territoriales. También considera los montos de recursos financieros asignados por el PEF 2002 y por los distintos organismos financieros internacionales para las entidades del s-SE, junto a las iniciativas mesoamericanas, respectivamente. Propone asimismo proyectos específicos que pueden operar en distintas subregiones identificadas.

Esta perspectiva, contenida en el documento de Avances y Perspectivas del PPP, revela un nuevo sentido y dimensión de las estrategias y proyectos que el Plan pretende realizar en la región Sur-Sureste de México. La parte medular son las denominadas estrategias regionales agrupadas en los rubros económico, social, ambiental y territorial. Para ello, propone lineamientos y criterios generales para la definición de proyectos. En esta etapa, los proyectos considerados asumen el carácter de emblemáticos (llamados así porque la mayoría de ellos sólo presentan propuestas generales y carecen de apoyo institucional y financiero real para su funcionamiento) y estaban considerados de manera parcial y fragmentada en el Programa de Egresos de la Federación. Se identificaron 15 proyectos generales, considerando que sus impulsores, además del Gobierno Federal, podrían ser los gobiernos estatales o algunos actores sociales relevantes.

La estrategia económica se orienta en dos sentidos: la ampliación y diversificación de las actividades económicas actuales y el desarrollo de nuevos recursos y capacidades. En el primer caso se consideran el despegue, fortalecimiento o creación de sinergias en los 
sectores de especialización de la región: agropecuario, turístico y algunas ramas manufactureras. El efecto territorial previsto pretende configurar corredores agroindustriales y de actividades diversificadas como circuitos turísticos y zonas industriales dinámicas susceptibles de articulación hacia corredores interregionales e interurbanos. En el segundo caso, se trata del desarrollo de capacidades que pueden detonar nuevas actividades o reposicionar zonas actualmente dinámicas. En esta vertiente se consideran actividades de investigación y desarrollo en biotecnología y químico-farmacéutica, en áreas con alta biodiversidad; acuacultura, en franjas costeras con aptitud; zonas con potencial para el desarrollo de plantaciones comerciales y polos de innovación, tecnopolos y clusters culturales en ciudades.

Pese a esta cartera de proyectos, lo cierto es que durante 2003 y 2004, el presupuesto asignado para el PPP, en el caso de México, fue orientado casi exclusivamente a consolidar los proyectos de infraestructura carretera en su porción del s-SE mexicano. En 2003 se asignó un presupuesto de 4329.4 millones de pesos, de los cuales $96 \%$ se incluyó en el programa carretero coordinado por la Secretaría de Comunicaciones y Transportes. Fuera de estos proyectos carreteros, los proyectos en otras áreas han sido casi nulos. Al respecto, sólo se podría mencionar el de transformación y trasmisión de energía eléctrica, los hospitales de medicina tradicional de Puebla y los recursos operativos de la oficina del PPP, cuya sede se trasladó en marzo de 2003 a la Secretaría de Relaciones Exteriores.

Para 2004, el PEF asignó un presupuesto de 861 millones de pesos a los proyectos del Programa de Desarrollo de la región Sur-Sureste y al PPP. Esta reducción resulta considerable, pues el presupuesto para este año es siete veces menor al de 2003 y cuatro veces menor al de 2002 en este rubro. En un contexto de ajustes fiscales y del gasto público para este año, la reducción de recursos financieros a los proyectos del S-SE repercutirá severamente en la concreción y los alcances previstos originalmente. Otro problema es que la estructura presupuestaria no cambió en términos de los rubros privilegiados; es decir, 95\% para segmentos carreteros y el restante se dividió en pocos proyectos: los de carácter eléctrico para Chiapas, una nueva central de abastos para ese mismo estado, la continuación del proyecto de vigilancia y control epidemiológico, acciones de saneamiento en Yucatán y recursos operativos para la coordinación del Plan y los vínculos con Centroamérica.

\section{El Plan Puebla-Panamá en su dimensión Centroamérica}

Las afinidades y problemáticas comunes entre el sureste mexicano y el istmo centroamericano fueron una de las justificaciones del Gobierno Federal en México para la adopción de un proyecto transterritorial con Centroamérica. Dichas afinidades están dadas en los rubros ambientales, histórico-culturales, condiciones de vida y migración. El territorio que incluye el PpP fue reconocido como la Región Mesoamericana, la cual representa $0.7 \%$ de la superficie de la tierra, alberga a 60 millones de habitantes, posee $10 \%$ de la biodiversidad

\section{DeSRarrollo}


global mediante diversos ecosistemas integrados y mantiene un grado importante de afinidad cultural e histórica (Presidencia de la República, 2001).

Como una iniciativa supranacional, la dimensión mesoamericana del PPP fue formalmente establecida mediante los acuerdos vertidos en reuniones presidenciales a mediados de 2001. Cabe destacar que, desde la década de los noventa, los países centroamericanos promovieron diversos esfuerzos para la definición de procesos de integración y agendas de desarrollo en diversos renglones. ${ }^{1}$

La relación más fructífera, en términos de colaboración reciente entre México y el bloque de países centroamericanos, ocurrió desde la década anterior. A partir de 1991, los presidentes de varios países centroamericanos y de México han celebrado de manera sistemática reuniones o cumbres denominadas Mecanismo de Diálogo y Concertación de Tuxtla o Cumbres de Tuxtla. Éstas habían permitido que los países centroamericanos y México adoptaran acuerdos de comercio, cooperación financiera, migración e infraestructura regional. Los temas, ahora replanteados en varias de las iniciativas del PPP, estaban presentes desde hace más de una década. Por ello, la Cumbre de Tuxtla es el mecanismo de más alto nivel, reconocido por los gobiernos de los países centroamericanos, con el cual se suscriben los términos y alcances de colaboración y articulación previstos del PPP. Lo novedoso

1 En 1994 se creó, por iniciativa de los presidentes centroamericanos, la llamada Agenda para la Competitividad y el Desarrollo Sostenible de Centroamérica hacia el Siglo XXI . Esta propuesta contó con el apoyo del Banco Centroamericano de Integración Económica y fue orientada al aprovechamiento de ventajas comparativas para la atracción de inversiones hacia la región. Dicho documento resulta ser la base más importante de las estrategias de inver sión y comercio, consideradas por los propios países centroamericanos.

En 1999, en el marco de los esfuerzos internacionales para la reconstrucción de los países afectados por el paso del huracán Mitch - ocurrido en 1998-, se constituyó el Grupo Consultivo Regional para la Transformación y M odernización de Centroamérica. A partir de las reuniones en Washington (1998) y Estocolmo (1999), los gobiernos centroamericanos y representantes de varios países suscribieron una declaración en la que se comprometieron a compartir la responsabilidad de la reconstrucción y de la transformación de la zona. Se estableció un proceso de consulta para el seguimiento del progreso de la iniciativa. Se integró un grupo de representantes de Alemania, Canadá, España, Estados Unidos de América y Suecia y, posteriormente, con la incorporación de Japón, se conformó lo que hoy se conoce como el G-6. Este grupo realizó también consultas con el Secretario General del Sistema de Integración Centroamericana (SICA) (Presidencia de la RepúblicaPNUD, 2001).

Posteriormente, durante la XX Cumbre de Presidentes Centroamericanos, realizada en 1999 en Guatemala, se analizó la posibilidad de realizar una reunión del G-6 en Madrid, España, con el objetivo de examinar las posibilidades de cooperación para apoyar a Centroamérica en su transformación y modernización. Se encomendó a la Secretaría General del sICA la elaboración del planteamiento centroamericano acerca de la transformación y modernización de la zona. Este documento fue resultado de un intenso proceso de consulta, en el que participaron representantes designados de los gobiernos del SICA y dela sociedad civil centroamericana, incluyen do empresarios einversionistas (Idem).

La iniciativa centroamericana fue presentada y discutida en la capital de España, en la reunión de enero de 2001 del G-6, por lo que es conocida como Planteamiento de Madrid. Esta iniciativa se propone profundizar procesos de integración, promoción de proyectos estratégicos y búsqueda de socios para la transformación y modernización de Centroamérica.

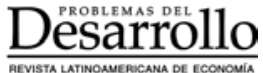


del Plan es, en todo caso, la prioridad e impulso que se otorga a varias iniciativas y sus fuentes de financiamiento.

La propuesta del PPP, en su versión México-Centroamérica, pareciera asumir por lo menos, un status complementario. Hoy se pretende acelerar las estrategias de comercio e inversión para la región, previamente diseñadas por los propios países involucrados, pues existe un fuerte interés para la implantación de megaproyectos que implican la atracción de inversiones productivas foráneas en varios sectores y la canalización de fuentes de financiamiento, mediante organismos como el Banco Mundial (BM), el Banco Interamericano de Desarrollo (BID) y el Banco Centroamericano de Integración Económica (BCIE). La dimensión transterritorial del PPP, la cual define la articulación real entre México y Centroamérica, está dada por las ocho Iniciativas Mesoamericanas y sus respectivos proyectos.

Para la instrumentación de estos proyectos se accedió a un fondo financiero, apoyado por el BID con poco más de 4000 millones de dólares, de los cuales 1512 millones (44.2\%) fueron destinados a México y el resto a las naciones centroamericanas. Cabe destacar que, al igual que la parte mexicana, los rubros privilegiados en las iniciativas mesoamericanas son los de infraestructura física, lo cual supone una lógica similar en los componentes que se han considerado como detonantes de los procesos de inversión-comercio en el conjunto de la región. $85 \%$ de los recursos están destinados a carreteras y $11 \%$ a la interconexión eléctrica. Las restantes seis iniciativas mantienen una posición accesoria en la estrategia mesoamericana, ya que solamente se les destina recursos equivalentes a $4 \%$ del conjunto de las iniciativas.

Dentro de las ocho iniciativas mesoamericanas, en 2003 se firmaron acuerdos de entendimiento en los renglones de desarrollo sustentable, prevención y mitigación de desastres, salud e integración vial, y quedaron en situación pendiente los restantes rubros. Debido al carácter vertical del PPP, la falta de participación y consensos con las comunidades de la región —no previstos por el proyecto-y las reacciones de movilización y foros sociales alternativos al PPP, recientemente el Plan consideró dos rubros que se suman a las ocho iniciativas mesoamericanas originalmente planteadas: el Programa de Información, Consulta y Participación y el Componente Indígena.

Por el impacto potencial en la región podemos destacar algunos de los proyectos que integran las iniciativas mesoamericanas. El Proyecto del Corredor Biológico Mesoamericano, el cual consiste en la creación de un sistema de áreas naturales protegidas — desde Oaxaca hasta Panamá- con la idea de que esta zona es una de las más importantes en el nivel mundial por su biodiversidad, presenta procesos de alto impacto ambiental y tiene potencial para desarrollar negocios en biotecnología.

El grueso del financiamiento y el eje de la articulación lo conforman los proyectos de interconexión eléctrica e integración vial. El primero considera la integración eléctrica

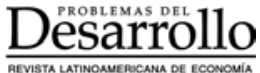


Cuadro 2

Iniciativas mesoamericanas: estimación de recursos

\begin{tabular}{lcc}
\hline Iniciativas mesoamericanas & Millones de dólares & Porcentaje \\
\hline Desarrollo sustentable & 16.5 & 0.41 \\
Desarrollo humano & 31.5 & 0.78 \\
Prevención y mitigación de desastres & 27.0 & 0.67 \\
Turismo & 51.7 & 1.28 \\
Facilitación del intercambio comercial & 23.5 & 0.58 \\
Integración vial & 420.6 & 85.2 \\
Interconexión energética & 445.7 & 11 \\
Telecomunicaciones & 1.2 & 0.29 \\
Gran total & 4017.7 & 100.00 \\
\hline
\end{tabular}

* \$1 512.8 millones de dólares corresponden a México (44.2\%).

Fuente: Presidencia de la República-BID (2001).

Belice-Guatemala y México-Guatemala, así como el Sistema de Interconexión Eléctrica para Centroamérica (SIEPAC), que permitirá concretar un mercado eléctrico centroamericano. Los proyectos de integración vial consideran básicamente el corredor del Pacífico y el del Atlántico, para reforzar la articulación de las capitales y ciudades más importantes de los países centroamericanos. En contraste, se advierte un avance limitado o incierto en proyectos de carácter social (desarrollo humano) y en proyectos económicos. Por este hecho, consideramos que los impactos del PPP serán principalmente de corto plazo y podrían reflejarse en una mayor integración regional de tipo transterritorial en ciertos rubros, dado el énfasis puesto en la accesibilidad y vías de comunicación y el mercado eléctrico regional. Ello puede ser una condición incipiente para lograr la atracción de capitales externos a la región, mejoramiento relativo de la infraestructura regional mediante la obra pública y mayor especialización de los mercados de productos y servicios específicos, pero que, de no tener continuidad y consolidación en el nivel local, podrían representar un nuevo fracaso en estos esfuerzos de planeación regional en México.

Un proyecto transterritorial como el PPP no plantea necesariamente un sentido de complementariedad interregional entre los diferentes países, ya que algunos de éstos muestran un perfil productivo similar en el renglón de bienes agropecuarios y manufacturas de exportación, así como de recursos naturales y escénicos potencialmente aprovechables. Esto significa nuevos riesgos por el posible incremento de los niveles de competencia entre los países involucrados en la propuesta del PPP. El riesgo sería todavía mayor para México, pues en la perspectiva de concretar las negociaciones del Tratado de Libre Comercio Estados Unidos-Centroamérica (CAFTA, por su siglas en inglés), nuestro país perdería el trato preferencial en productos exportables que actualmente incursionan al mercado norteamericano. 
Cuadro 3

Situación que guardan las iniciativas mesoamericanas

\begin{tabular}{|c|c|c|}
\hline $\begin{array}{c}\text { Iniciativa } \\
\text { mesoamericana }\end{array}$ & Proyectos & Avance \\
\hline 1. Desarrollo humano & $\begin{array}{l}\text { 1. Educación Mesoamericana } \\
\text { 2. Proyecto Regional de Salud y Desarrollo Humano } \\
\text { 3. Sistema de Demanda y Servicios de Capacitación } \\
\text { para Mesoamérica } \\
\text { 4. Sistema de Información Estadístico sobre Migraciones }\end{array}$ & $\begin{array}{l}\text { Memorando de entendi- } \\
\text { miento en salud, } \\
\text { septiembre de } 2003\end{array}$ \\
\hline 2. Desarrollo sustentable & $\begin{array}{l}\text { 1. Apoyo a la Iniciativa de Desarrollo Sostenible } \\
\text { 2. Corredor Biológico Mesoamericano } \\
\text { 3. Programa Mesoamericano de Desarrollo Sostenible } \\
\text { de Recursos Naturales en Áreas Multinacionales } \\
\text { 4. Programa Mesoamericano de Sistemas de Gestión } \\
\text { Ambiental (PROSIGA) }\end{array}$ & $\begin{array}{l}\text { Memorando suscrito el } 2 \\
\text { de junio de } 2003 \text { en } \\
\text { Washington D.C. }\end{array}$ \\
\hline $\begin{array}{l}\text { 3. Prevención y miti- } \\
\text { gación de desastres } \\
\text { naturales }\end{array}$ & $\begin{array}{l}\text { 1. Concesión Pública para Prevención de Desastres } \\
\text { 2. Desarrollo de Mercado de Seguros contra Riesgos } \\
\text { de Catástrofe } \\
\text { 3. Información Hidrometereológica para la } \\
\text { Competitividad }\end{array}$ & $\begin{array}{l}\text { Firma de Memorando el } 13 \\
\text { de noviembre de } 2003 \text { en } \\
\text { la Ciudad de Panamá }\end{array}$ \\
\hline 4. Turismo & $\begin{array}{l}\text { 1. Certificación de Sustentabilidad Turística } \\
\text { 2. Desarrollo de Circuitos Turísticos Integrales en la } \\
\text { Región Mesoamericana } \\
\text { 3. Ecoturismo } \\
\text { 4. Fortalecimiento de la Seguridad Aeroportuaria } \\
\text { 5. Implementación de Cuentas Satélites de Turismo }\end{array}$ & En proceso \\
\hline $\begin{array}{l}\text { 5. Facilitación del } \\
\text { intercambio comercial }\end{array}$ & $\begin{array}{l}\text { 1. Armonización de Normas Fito y Zoosanitarias } \\
\text { 2. Modernización de Aduanas y Pasos Fronterizos } \\
\text { 3. Programa de Cooperación Técnica en el Sector } \\
\text { Financiero } \\
\text { 4. PYME }\end{array}$ & $\begin{array}{l}\text { - Curso-taller acerca de } \\
\text { Financiamiento de Proyec- } \\
\text { tos del PPP y Cooperación } \\
\text { Financiera en Mesoamérica } \\
\text { - Aprobación de préstamo } \\
\text { para modernización de } \\
\text { aduanas y pasos fronterizos }\end{array}$ \\
\hline 6. Telecomunicaciones & $\begin{array}{l}\text { 1. AMI: Autopista Mesoamericana de la Información } \\
\text { 2. Marco Regulativo Regional } \\
\text { 3. Utilización Social de las TIC's: apoyo a la } \\
\text { Red Regional de Telecentros }\end{array}$ & Memorando por firmar \\
\hline
\end{tabular}


Continuación Cuadro 3

\begin{tabular}{|c|c|c|}
\hline $\begin{array}{c}\text { Iniciativa } \\
\text { mesoamericana }\end{array}$ & Proyectos & Avance \\
\hline 7. Integración energética & $\begin{array}{l}\text { 1. Área de Actividad de Promoción de las Energías } \\
\text { Renovables y Uso de los Biocombustibles } \\
\text { 2. Área de Electrificación y Energización Rural } \\
\text { 3. Conexión Guatemala-Belice } \\
\text { 4. Interconexión México-Guatemala } \\
\text { 5. Sistema de Interconexión Eléctrica de los Países de } \\
\text { América Central (SIEPAC) }\end{array}$ & $\begin{array}{l}\text { - Estudios para la confor- } \\
\text { mación del Mercado Eléc- } \\
\text { trico Regional } \\
\text { - Aprobación del BID prés- } \\
\text { tamo para Guatemala: cons- } \\
\text { trucción de } 71 \mathrm{~km} \text { de líneas } \\
\text { para interconexión México- } \\
\text { Guatemala. Estudios de Im- } \\
\text { pacto ambiental en } 32 \mathrm{~km} \\
\text { de la parte mexicana }\end{array}$ \\
\hline 8. Integración Vial & $\begin{array}{l}\text { 1. Armonización de Regulaciones y Normas Técnicas } \\
\text { 2. RICAM: Corredor del Atlántico } \\
\text { 3. RICAM: Corredor del Pacífico (Puebla-Panamá) } \\
\text { 4. RICAM: Ramales y Conexiones Regionales } \\
\text { Complementarios }\end{array}$ & $\begin{array}{l}\text { Memorando firmado el } \\
28 \text { de junio } 2003 \\
\text { en Mérida, México }\end{array}$ \\
\hline
\end{tabular}

Fuente: elaboración propia a partir de Iniciativas Mesoamericanas, Avance 2003.

Plan Puebla-Panamá. Iniciativas Mesoamericanas, Banco de Desarrollo Interamericano. http://www.iadb.org/ ppp/project/

\section{Conclusiones}

El análisis de iniciativas como el PPP y su incidencia en los procesos de desarrollo regional permiten evaluar el impacto de una nueva generación de políticas públicas que se proponen insertar y reactivar regiones tradicionalmente marginadas en los procesos de acumulación de capital, tomando en cuenta las posibles oportunidades que se generan en los procesos mundiales de inversión y comercio.

Para incorporar nuevas regiones en dicho esquema, resulta fundamental modificar la geografía de la producción, las inversiones y los mercados de trabajo, en aras de revalorar espacios atractivos para el capital. En consecuencia, el Estado asume nuevos papeles en la política económica regional que lo llevan a concentrar sus esfuerzos y recursos hacia determinados territorios, privilegiando los factores exógenos que permitan aprovechar las posibles ventajas del entorno local en las inversiones y el comercio.

Las estrategias de incorporación de los territorios, como la que considera el PPP, podrían ser múltiples, ya sean espacios de inversión para el aprovechamiento de los recursos regionales de alto valor o territorios para consolidar cadenas de producción mundiales —-mediante la generación de bienes y servicios de alto valor por parte de empresas trasnacionalestoda vez que no ha sido posible un aprovechamiento mayor por parte de los grupos de capitales nacionales y regionales.

Sin embargo, a tres años de inicio del PPP, los impactos resultan limitados, producto de los escasos recursos presupuestales y de su orientación hacia rubros de infrestructura en 
transportes y energética. Ello supone privilegiar en esta etapa procesos de accesibilidad y conectividad interregionales: una circunstancia necesaria, pero todavía insuficiente para generar condiciones de inversión regionales, aunque ello significa un mejoramiento rela-tivo de la infraestructura regional mediante la obra pública en este momento.

No obstante, las políticas públicas de desarrollo regional orientadas en este sentido resultan limitadas, pues se requiere de una base económica previamente construida y de plazos mucho más largos que le permitan absorber favorablemente los procesos de inversión y comercio, fomentar una mayor especialización de los mercados de productos y servicios específicos regionales, y generar condiciones de bienestar social y desarrollo endógeno y sostenido.

Por lo tanto, el reto para una región como la Sur-Sureste, con el atraso secular de desarrollo y menor grado de integración a la economía nacional e internacional, será el de establecer capacidades productivas, tecnológicas y sociales que le permitan en el futuro absorber positivamente los impactos de los proyectos de inversión y comercio emanados de políticas y planes de esta naturaleza.

La situación de atraso en el conjunto del área de influencia del PPP y las condiciones similares en algunos sectores productivos, más bien aparecen como factores que podrían fomentar una mayor competencia interregional, más que la integración y complementación a partir de esta iniciativa. Ello hace cuestionable el éxito de esta estrategia, al menos desde los objetivos que se propone en cuanto a generar desarrollo y abatir rezagos.

\section{Bibliografía}

Alburquerque, Francisco, "Ajuste Estructural e Iniciativa de Desarrollo Local", Revista Comercio Exterior, vol. 51, núm. 8, México, 2001.

Allen, Scott, "Regiones Urbano - Globales. Dilemas de la Planeación y de Política en un Mundo Neoliberal", en Jorge Basave et al. (coordinadores), Globalización y Alternativas Incluyentes para el Siglo XXI, México, IIEC, CRIM, Facultad de Economía, DGAPA, UNAM, Miguel Ángel Porrúa, 2002.

Álvarez Béjar, Alejandro (coordinador), "El Plan Puebla-Panamá ¿Para el desarrollo regional o de un enclave transnacional?", en Memoria, México, núm. 148, junio, 2001.

Álvarez Alejandro, A. Barreda y A. Bartra, Economía política del Plan Puebla Panamá, Editorial ITACA, México, 2001.

Arce, Randall y Greivin Hernández, Centroamérica en el contexto del Plan Puebla-Panamá, Centro Internacional de Política Económica para el Desarrollo Económico, 2002.
Arroyo Picard, Alberto; Jorge Calderón et al., (coordinadores), Área de Libre Comercio de las Américas. Respuesta social a la agenda corporativa, México, IERD-Fundación Friedrich Ebert, 2001.

Bailey, John, Impactos del TLC en México y Estados Unidos. Efectos subregionales del comercio y la integración económica, México, Miguel Ángel Porrúa, Georgetown University, FLACSO, 2003.

Benko, Georges, "Desarrollo Regional. Revisando las dos últimas décadas", en Leticia Campos (coordinadora), La realidad económica actual y las corrientes teóricas de su interpretación, México, IIEC-UnAm, Plaza y Valdés, 2002.

BCIE-BID-CEPAL, Plan Puebla-Panamá. Iniciativas Mesoamericanas y Proyectos, Grupo Técnico Interinstitucional para el Plan Puebla-Panamá, Banco Interamericano de Integración Económica, Banco Interamericano de Desarrollo, Comisión Económica de América Latina con el apoyo del INCAE, El Salvador, 2001. 
Conectividad de la propuesta regional de modernización y transformación de Centroamérica y del Plan Puebla Panamá, Banco Interamericano de Integración Económica, Banco Interamericano de Desarrollo, Comisión Económica de América Latina, 2001a.

BID, Las economías de los países Centroamericanos y República Dominicana: Evolución y desafios de largo plazo, Washington, D.C., Serie de Estudios Económicos y Sectoriales, noviembre, 2002.

Biehl, Dieter, "Las infraestructuras y el desarrollo regional", Papeles de Economía Española, Madrid, Fundación fondo para la investigación económica y social, 1998.

Boisier, Sergio, Modernidad y Territorio, Santiago de Chile, Instituto Latinoamericano y del Caribe de Planificación Económica y Social, CEPAL, 1996.

Briceño R., José, "El Modelo TLCAN de Investigación y Negociaciones del ALCA", Revista Problemas del Desarrollo, vol. 32, núm. 126, México, IIECUNAM, 2001.

Caravaca, Inmaculada, "Los nuevos espacios ganadores y emergentes", Revista Eure, vol. xxIv, núm. 79, Santiago de Chile, 1998.

Consejo Consultivo del Sistema de Integración Centroamericano (CC-SICA), Declaración del CCSICA sobre el Plan Puebla-Panamá, www.codehuca. or.cr/centroamericaaldia.htm, 2001.

Consejo Indígena de Centroamérica, Resultados preliminares del foro de consulta sobre el Plan PueblaPanamá, presentados en la Cumbre de Presidentes en Mérida, Yucatán, http://www. redindigena.net/articulos/06-28ppp.html, 2002

Dabat, Alejandro, "Los Procesos de Integración Regional en el Mundo y sus Impactos Internacionales", en Lucía González y Serafín Maldonado (coordinadores), La globalización y sus Impactos Socioterritoriales, México, Universidad de Guadalajara, 2003.

Dávila, Enrique, Georgina Kessel y Santiago Levy, "El Sur también existe: ensayo sobre el desarrollo regional de México", Economía Mexicana, vol. XI, núm. 2, México, CIDE, 2002.

Delgadillo, Javier, Felipe Torres y José Gasca, El Desarrollo Regional de México en el Vértice de Dos Milenios, IIEC-Unam, México, Porrúa, 2001.

Ferreira, Héctor, "Continuidad y Cambio en la política regional", Ponencia presentada en las Sextas Jornadas Multidisciplinarias del CRIM-UNAM. Cuernavaca, Morelos, 2003.
Furió Blasco, E., Desarrollo territorial y procesos de innovación. Les Milieux innovateurs, París, 1996.

Guerra Borges, Alfredo, "Variaciones sobre el futuro de la globalización", en Globalización y Alternativas Incluyentes para el Siglo XXI, en Jorge Basave et al. (coordinadores), México, IIEC, CRIM, Facultad de Economía, DGAPA, UNAM, Miguel Ángel Porrúa, 2002.

Harris, Nigel, "El Estado Nación ante el Proceso de Globalización y sus Impactos Socioterritoriales", en Lucía González y Serafín Maldonado, (coordinadores), La Globalización y sus Impactos Socioterritoriales, México, Universidad de Guadalajara, 2003.

Helmsing, Bert A.H.J., "Teorías del desarrollo industrial regional y políticas de segunda y tercera generación”, en Estudios Urbano Regionales, vol. 25, núm. 75, Santiago de Chile, septiembre, 1999.

Indacochea, Alejandro, "La promoción país y el desarrollo de las ventajas competitivas", en Revista Comercio exterior, vol. 51, núm. 8, México, 2001.

Ornelas Delgado, Jaime, "El Plan Puebla-Panamá y la Globalización Neoliberal", Revista Aportes, año viI, núm. 21, México, Facultad de Economía, Benemérita Universidad Autónoma de Puebla, 2001.

Moncayo J. Edgar, Nuevos enfoques teóricos, evolución de las políticas regionales e impacto territorial en la globalización, Santiago de Chile ILPES-CEPAL, Serie Gestión Pública núm. 27, 2002.

Presidencia de la República, Plan Puebla-Panamá, México, Síntesis del Proyecto, México, Presidencia de la República. 2001.

_, Plan Puebla-Panamá, Capítulo México, Documento Base México, México, Presidencia de la República, 2001a.

, Programa de las Naciones Unidas para el Desarrollo, Proyecto Plan Puebla-Panamá. Informe de avances y perspectivas (CD), México, 2002.

Pujadas, Romá y Jaume Font, Ordenación y planificación territorial, Madrid, Editorial Síntesis, 1998.

Rajchenberg, Enrique y Catherine Héau-Lambert, En la antesala del Plan Puebla-Panamá: Tehuantepec en el Siglo XIX. Revista Chiapas núm. 14, México, ERA-IIEC-UNAM, 2002.

Rudiño, Lourdes Edith, "Plan Puebla-Panamá, megaproyecto regional", Reportaje de $E l$ Financiero, 19 de febrero del 2001.

\section{Desarrollo}

